\title{
artigo
}

\section{Segurança do paciente como práxis da atenção à saúde: uma reflexão teórica sobre a literatura}

\author{
Patients safety as practices of health care: a theoretical reflection on literature
}

La seguridad del paciente como prática de la atención sanitaria: uma reflexión teórica sobre la literatura

\begin{abstract}
RESUMO
Objetivo: Analisar, a partir de uma reflexão teórica, o contexto da segurança do paciente na atenção à saúde. Método: Trata-se de um estudo qualitativo, descritivo do tipo teórico-reflexivo desenvolvido a partir da abordagem de revisão narrativa com artigos científicos nacionais e internacionais disponiveis nas bases de dados LILACS, BDENF, SciELO e MEDLINE. Resultados: 0 conceito de segurança do paciente tem evoluído entre os profissionais de saúde, bem como em outras áreas das instituições de saúde hospitalares, inclusive na alta liderança, o que estimula o envolvimento da instituição como um todo. No Brasil, ainda há uma elevada incidência de ocorrência de eventos adversos que poderiam ser evitados Conclusão: Tem-se a necessidade de ampliar as investigações sobre eventos adversos em outros níveis de atenção quanto a adoção de estratégias relacionadas à segurança do paciente precisa ser melhor desenvolvida.
\end{abstract}

DESCRITORES: Segurança do paciente; Atenção primária à saúde; Qualidade da assistência à saúde.

\section{ABSTRACT}

Objective: To analyze, from a theoretical reflection, the context of patient safety in health care. Method: This is a qualitative, descriptive study of the theoretical-reflective type developed from the narrative review approach with national and international scientific articles available in the LILACS, BDENF, SciELO and MEDLINE databases. Results: The concept of patient safety has evolved among health professionals, as well as in other areas of hospital health institutions, including senior leadership, which encourages the involvement of the institution as a whole. In Brazil, there is still a high incidence of the occurrence of adverse events that could be avoided. Conclusion: There is a need to expand investigations on adverse events at other levels of care regarding the adoption of strategies related to health safety patient needs to be better developed.

DESCRIPTORS: Patient safety; Primary health care; Quality of health care.

\section{RESUMEN}

Objetivo: Analizar, desde uma reflexión teórica, el contexto de la seguridade del paciente em el cuidado de la salud. Método: Se trata de um estúdio cualitativo, descriptivo de tipo teórico-reflexivo desarrollado a partir del enfoque de revisión narrativa com artículos científicos nacionales e internacionales disponibles em las bases de datos LILACS, BDENF, SciELO y MEDLINE. Resultados: El concepto de seguridade del paciente há evolucionado entre los profesionales de la salud, así como em otras áreas de las instituciones de salud hospitalaria., incluyendo la alta dirección, lo que incentiva la participación de la intitución em su conjunto. Em Brasil, tadavía existe uma alta incidência de eventos adversos que podrían evitarse. Conclusión: Es necessário ampliar las investigaciones sobre eventos adversos em otros niveles de atención, ya que la adopción de estratégias relacionadas com la seguridade del paciente debe desarrollarse más.

DESCRIPTORES: Seguridad del paciente; Atención primaria de salud; Calidad de la atención de salud.

RECEBIDO EM: 06/03/2021 APROVADO EM: 05/04/2021

\section{Ricardo Saraiva Aguiar}

Universidade Católica de Brasilia (UCB). Escola de Saúde e Medicina, Programa de Pós-Graduação em Gerontologia, Brasilia-DF, Brasil. ORCID: 0000-0003-0335-2194

\section{Henrique Salmazo da Silva}

Universidade Católica de Brasilia (UCB). Escola de Saúde e Medicina, Programa de Pós-Graduação em Gerontologia, Brasilia-DF, Brasil. ORCID: 0000-0002-3888-4214 


\section{INTRODUÇÃO}

A segurança do paciente tem sido um dos grandes alvos de discussão e preocupação nas últimas décadas pelos profissionais de saúde, gestores e pesquisadores devido ao impacto que os eventos adversos podem ocasionar na qualidade da assistência à saúde ${ }^{1-2}$.

Essa passou a estar mais presente nas pautas das agendas dos serviços de saúde públicos e privados após a publicação em 1999 do relatório norte-americano "Errar é humano: construindo um sistema de saúde mais seguro" do Instituto de Medicina $(\mathrm{IOM})^{3-5}$.

O documento apresenta os resultados do estudo de prontuários que apontou a ocorrência de iatrogenias em um pouco mais de $3 \%$ do total analisado. Além disso, foram documentados de 44 a $98 \mathrm{mil}$ óbitos de pacientes por ano nos Estados Unidos da América (EUA). Grande parcela deles poderia ter sido evitado com a adoção de práticas voltadas a promoção de um cuidado seguro ${ }^{3}$.

Dessa maneira, após a veiculação desses dados, as agências sanitárias despertaram maior interesse pelo tema, o que possibilitou adotar medidas para melhoria da qualidade dos serviços de saúde e para redução da incidência de eventos adversos ${ }^{6}$.

Para o alcance desse cuidado, dito como seguro, as instituições de saúde têm se esforçado em melhorar os processos de cuidado oferecidos aos usuários, reconhecendo em primeira instância a necessidade de se estabelecer a cultura de segurança do paciente como rotina do serviço de saú$\mathrm{de}^{7-8}$. A compreensão desse cenário pela instituição a partir da análise da cultura de segurança torna-se, dessa forma, o ponto de partida para traçar ações em prol de mudanças para a redução dos incidentes e a garantia de cuidados de saúde seguros ${ }^{9-10}$.

Diante dessa realidade, a avaliação da segurança é vista como o ponto de partida para compreender o cenário atual e iniciar o planejamento de ações que busquem mudanças para reduzir a incidência de eventos adversos ${ }^{11}$. Ela permite identificar e gerir prospectivamente questões relevantes à segurança nas rotinas de trabalho, com vistas a garantir cuidados de saúde seguros na prática geral ${ }^{12}$.

Portanto, este artigo tem o objetivo de analisar, a partir de uma reflexão teórica, o contexto da segurança do paciente na atenção à saúde.

\section{MÉTOdOS}

Trata-se de um estudo qualitativo, descritivo do tipo teórico-reflexivo desenvolvido a partir da abordagem de revisão narrativa com artigos científicos nacionais e internacionais que abordam sobre o contexto da segurança do paciente na atenção à saúde.

Realizou-se, para a busca das produções, a seguinte pergunta de pesquisa: "Qual a produção, na literatura, sobre a segurança do paciente no contexto da atenção à saúde?". Selecionavam-se, assim, os artigos que abordavam a segurança do paciente e atenção à saúde.

Os artigos foram pesquisados no período de janeiro a março de 2020 por meio das bases de dados Literatura Latino-Americana e do Caribe em Ciências da Saúde (LILACS), Medical Literature Analysis and Retrieval System Online (MEDLINE), Base de Dados em Enfermagem (BDENF) e Scientific Eletronic Library Online (SciELO), refinando a busca para o período de 2009 a 2020 e utilizando os seguintes critérios de inclusão: artigos disponíveis de forma online; nos idiomas português, inglês ou espanhol; e pesquisas originais ou de revisão de literatura.

Dividiu-se o estudo em etapas: pesquisa dos artigos nas bases de dados; leitura dos títulos e dos resumos para verificar a convergência do material à temática de estudo e aos critérios de inclusão; leitura do artigo na íntegra; busca e leitura dos estudos originais encontrados por meio das referências finais dos artigos oriundos da busca nas bases de dados. Realizaram-se, após todas as leituras, a compilação dos materiais, seguida da análise e identificação do contexto da segurança do paciente na atenção à saúde para a reflexão e, finalmente, a elaboração das sínteses reflexivas do estudo.

Com os artigos selecionados, utilizou-se a síntese narrativa para análise e discussão dos dados.

\section{RESULTADOS}

Foram utilizados 13 artigos nesta revisão narrativa (Quadro 1), sendo interpretados e sintetizados os resultados através de uma comparação dos dados evidenciados na análise dos artigos.

\section{DISCUSSÃO}

A satisfação dos pacientes com a atenção à saúde tem sido questionada há mui-

Quadro 1 - Distribuição dos artigos de acordo com o periódico, ano de publicação, autor(es), título, delineamento e resultados. Brasília, Distrito Federal, Brasil, 2021.

\begin{tabular}{|c|c|c|c|c|c|}
\hline & $\begin{array}{l}\text { PERIÓDICO } \\
\text { E ANO DE } \\
\text { PUBLICAÇÃO }\end{array}$ & AUTOR(ES) & TÍTULO & $\begin{array}{l}\text { DELINEA- } \\
\text { MENTO }\end{array}$ & RESULTADOS \\
\hline 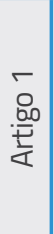 & $\begin{array}{c}\text { Revista Bioéti- } \\
\text { ca, } 2018\end{array}$ & $\begin{array}{c}\text { Romero MP, } \\
\text { González RB, Calvo } \\
\text { MSR, Fachado AA }\end{array}$ & $\begin{array}{l}\text { A segurança do pa- } \\
\text { ciente, qualidade do } \\
\text { atendimento e ética dos } \\
\text { sistemas de saúde }\end{array}$ & $\begin{array}{c}\text { Reflexão } \\
\text { Teórica }\end{array}$ & $\begin{array}{l}\text { As obrigações éticas em torno da prevenção de } \\
\text { erros médicos e da promoção da segurança do } \\
\text { paciente são configuradas pelos quatro princípios } \\
\text { da bioética. Essas obrigações não são apenas de- } \\
\text { veres pessoais de cada um dos profissionais, mas } \\
\text { também das instituições de saúde como um todo. }\end{array}$ \\
\hline
\end{tabular}




\section{artigo}

Aguiar, R.S.; Salmazo da Silva, H

Segurança do paciente como práxis da atenção à saúde: uma reflexão teórica sobre a literatura

\begin{tabular}{|c|c|c|c|c|c|}
\hline 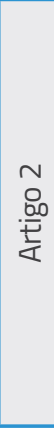 & $\begin{array}{l}\text { Cadernos de } \\
\text { Saúde Pública, } \\
2018\end{array}$ & $\begin{array}{c}\text { Rech RS, Hugo } \\
\text { FN, Giordani JMA, } \\
\text { Passero LG Hilgert } \\
\text { JB }\end{array}$ & $\begin{array}{l}\text { Contextual and } \\
\text { individual factors } \\
\text { associated with } \\
\text { dissatisfaction with } \\
\text { public emergency } \\
\text { health services in } \\
\text { Brazil, 2011-2012 }\end{array}$ & $\begin{array}{l}\text { Transversal } \\
\text { multinivel }\end{array}$ & $\begin{array}{c}\text { A prevalência de insatisfação percebida } \\
\text { era } 48,1 \% \text {. As variáveis que mantiveram a } \\
\text { associação significativa com o desfecho foram: } \\
\text { idade }>20 \text { anos, escolaridade } \geq 16 \text { anos, Região } \\
\text { Centro-Oeste, demandas não atendidas, tempo } \\
\text { de espera mais longo e acesso ao atendimento } \\
\text { de emergência em serviços de atenção primária. } \\
\text { A prevalência da insatisfação percebida está } \\
\text { relacionada predominantemente ao tempo de } \\
\text { espera e ao tempo necessário para resolver a } \\
\text { demanda. }\end{array}$ \\
\hline 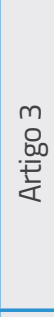 & $\begin{array}{l}\text { Int J Nurs } \\
\text { Stud, } 2016\end{array}$ & $\begin{array}{l}\text { Rosse FV, Bruijne } \\
\text { M, Suurmond J, } \\
\text { Essink-Bot ML, } \\
\text { Wagner C }\end{array}$ & $\begin{array}{l}\text { Language barriers and } \\
\text { patient safety risks in } \\
\text { hospital care: A mixed } \\
\text { methods study }\end{array}$ & Misto & $\begin{array}{c}\text { Situações no atendimento hospitalar em } \\
\text { que uma barreira de linguagem ameaçava a } \\
\text { segurança do paciente incluíam tarefas diárias } \\
\text { de enfermagem, ou seja, administração de } \\
\text { medicamentos, controle da dor, gerenciamento } \\
\text { do equilíbrio de fluídos) e interação médico-pa- } \\
\text { ciente em relação ao diagnóstico, comunicação } \\
\text { de risco e situações agudas. }\end{array}$ \\
\hline $\begin{array}{l}\text { 走 } \\
\text { 尊 } \\
\end{array}$ & $\begin{array}{c}\text { Revista } \\
\text { Gaúcha de } \\
\text { Enfermagem, } \\
2019\end{array}$ & $\begin{array}{l}\text { Reis GAX, Oliveira } \\
\text { JLC, Ferreira AMD, } \\
\text { Vituri DW, Marcon } \\
\text { SS, Matsuda LM }\end{array}$ & $\begin{array}{l}\text { Dificuldades para } \\
\text { implantar estratégias de } \\
\text { segurança do paciente: } \\
\text { perspectivas de enfer- } \\
\quad \text { meiros gestores }\end{array}$ & Qualitativo & $\begin{array}{l}\text { Os enfermeiros elencaram a insuficiência de } \\
\text { pessoal de enfermagem, o déficit no apoio da alta } \\
\text { gestão e a falta de adesão dos trabalhadores da } \\
\text { assistência como dificuldades importantes para } \\
\text { a implantação de estratégias de segurança do } \\
\text { paciente. }\end{array}$ \\
\hline 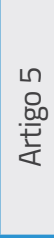 & $\begin{array}{l}\text { Ciência \& Saú- } \\
\text { de Coletiva, } \\
2013\end{array}$ & $\begin{array}{c}\text { Reis CT, Martins M, } \\
\text { Laguardia J }\end{array}$ & $\begin{array}{l}\text { A segurança do paciente } \\
\text { como dimensão da } \\
\text { qualidade do cuidado de } \\
\text { saúde: um olhar sobre a } \\
\text { literatura }\end{array}$ & $\begin{array}{l}\text { Revisão de } \\
\text { literatura }\end{array}$ & $\begin{array}{c}\text { A investigação sobre a segurança do paciente ain- } \\
\text { da não possui o benefício de ter suas abordagens } \\
\text { bem estabelecidas; e ainda, que múltiplas barreiras } \\
\text { e desafios precisam ser enfrentados na concepção } \\
\text { de delineamentos de estudos e na utilização de } \\
\text { técnicas de pesquisa. }\end{array}$ \\
\hline 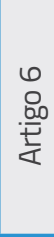 & $\begin{array}{c}\text { Saúde em } \\
\text { Debate, } 2016\end{array}$ & $\begin{array}{c}\text { Silva AT, Alves MG, } \\
\text { Sanches RS, Terra } \\
\text { FS, Resck ZMR }\end{array}$ & $\begin{array}{l}\text { Assistência de enfer- } \\
\text { magem e o enfoque da } \\
\text { segurança do paciente } \\
\text { no cenário brasileiro }\end{array}$ & $\begin{array}{c}\text { Revisão } \\
\text { integrativa } \\
\text { da literatura }\end{array}$ & $\begin{array}{l}\text { Identificou-se a existência de baixo conhecimento } \\
\text { dos profissionais de saúde sobre eventos adversos } \\
\text { e como notificá-los, medo dos profissionais de } \\
\text { saúde em expor os erros devido à política de puni- } \\
\text { ção das instituições e baixa adesão da técnica de } \\
\text { higienização das mãos. }\end{array}$ \\
\hline 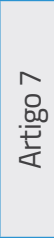 & $\begin{array}{l}\text { Cadernos de } \\
\text { Saúde Pública, } \\
2016\end{array}$ & $\begin{array}{l}\text { Gama ZAS, Satur- } \\
\text { no-Hernández PJ, } \\
\text { Ribeiro DNC, Frei- } \\
\text { tas MR, Medeiros } \\
\text { PI, Batista AM et al }\end{array}$ & $\begin{array}{l}\text { Desenvolvimento e } \\
\text { validação de indicado- } \\
\text { res de boas práticas de } \\
\text { segurança do paciente: } \\
\text { Projeto ISEP-Brasil }\end{array}$ & $\begin{array}{l}\text { Validação } \\
\text { de instru- } \\
\text { mentos de } \\
\text { medida }\end{array}$ & $\begin{array}{c}\text { Aprovaram-se } 75 \text { indicadores de boas práticas } \\
\text { (39 de estrutura; } 36 \text { de processo) para } 31 \text { das } 34 \\
\text { recomendações. Os indicadores foram conside- } \\
\text { rados válidos, confiáveis e úteis para o monito- } \\
\text { ramento da segurança do paciente em hospitais } \\
\text { brasileiros. }\end{array}$ \\
\hline $\begin{array}{l}\infty \\
0 \\
0.0 \\
.00 \\
.01\end{array}$ & $\begin{array}{l}\text { Revista } \\
\text { Brasileira de } \\
\text { Terapia Inten- } \\
\text { siva, } 2009\end{array}$ & $\begin{array}{c}\text { Beccaria LM, Perei- } \\
\text { ra RAM, Cotrin LM, } \\
\text { Lobo SMA, Trajano } \\
\text { DHL }\end{array}$ & $\begin{array}{l}\text { Eventos adversos na } \\
\text { assistência de enferma- } \\
\text { gem em uma unidade de } \\
\text { terapia intensiva }\end{array}$ & $\begin{array}{l}\text { Quantita- } \\
\text { tivo }\end{array}$ & $\begin{array}{l}\text { Foram registrados } 550 \text { eventos adversos rela- } \\
\text { cionados aos cinco certos na administração de } \\
\text { medicamentos; à medicações não administra- } \\
\text { das; às anotações inadequadas da medicação; } \\
\text { à falhas na instalação de drogas em bomba } \\
\text { de infusão; à não realização da inalação; ao } \\
\text { manuseio incorreto de seringas e agulhas; aos } \\
\text { procedimentos de enfermagem não realizados; } \\
\text { ao manuseio incorreto de artefatos terapêuticos } \\
\text { e diagnósticos; aos alarmes dos equipamentos } \\
\text { utilizados de maneira incorreta; e à falhas nas } \\
\text { anotações de enfermagem. }\end{array}$ \\
\hline
\end{tabular}




\begin{tabular}{|c|c|c|c|c|c|}
\hline $\begin{array}{l}a \\
0 \\
.0 \\
.00 \\
\frac{1}{2}\end{array}$ & $\begin{array}{l}\text { Texto \& } \\
\text { Contexto } \\
\text { Enfermagem, } \\
2017\end{array}$ & $\begin{array}{c}\text { Reis GAX, } \\
\text { Hayakawa LY, Mu- } \\
\text { rassaki ACY, Mat- } \\
\text { suda LM, Gabriel } \\
\text { CS, Oliveira MLF }\end{array}$ & $\begin{array}{l}\text { Implantação das estra- } \\
\text { tégias de segurança do } \\
\text { paciente: percepções de } \\
\text { enfermeiros gestores }\end{array}$ & Qualitativo & $\begin{array}{l}\text { O processo de implantação das estratégias de } \\
\text { segurança do paciente é percebido de forma con- } \\
\text { traditória pelos participantes, mas, apesar disso, } \\
\text { emitiram sentimentos de satisfação. }\end{array}$ \\
\hline 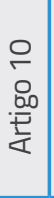 & $\begin{array}{l}\text { BMJ Qual Saf, } \\
2013\end{array}$ & $\begin{array}{l}\text { Jha AK, Larizgoitia } \\
\text { I, Audera-Lopez C, } \\
\text { Prasopa-Plaizier N, } \\
\text { Waters H, Bates D }\end{array}$ & $\begin{array}{l}\text { The global burden of } \\
\text { unsafe medical care: } \\
\text { analytic modelling of } \\
\text { observational studies }\end{array}$ & $\begin{array}{l}\text { Estudo ob- } \\
\text { servacional }\end{array}$ & $\begin{array}{l}\text { Estima-se que haja } 421 \text { milhões de hospitaliza- } \\
\text { ções no mundo anualmente e aproximadamente } \\
42,7 \text { milhões de eventos adversos. Aproximada- } \\
\text { mente dois terços de todos os eventos adversos } \\
\text { acontecem em países de baixa e media renda. }\end{array}$ \\
\hline 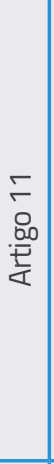 & $\begin{array}{l}\text { Revista da } \\
\text { Associação } \\
\text { Médica Brasi- } \\
\text { leira, } 2013\end{array}$ & $\begin{array}{l}\text { Mendes Júnior W, } \\
\text { Pavão ALB, Martins } \\
\text { M, Moura MLO, } \\
\text { Travassos C }\end{array}$ & $\begin{array}{l}\text { Características de even- } \\
\text { tos adversos evitáveis } \\
\text { em hospitais do Rio de } \\
\text { Janeiro }\end{array}$ & $\begin{array}{l}\text { Coorte re- } \\
\text { trospectivo }\end{array}$ & $\begin{array}{c}\text { Foram identificados } 65 \text { eventos adversos evitáveis } \\
\text { dos } 56 \text { pacientes que sofreram eventos adversos } \\
\text { evitáveis. As infecções associadas aos cuidados da } \\
\text { saúde representaram } 24,6 \% \text {; complicações cirúrgi- } \\
\text { cas e/ou anestésicas, } 20 \% \text {; danos decorrentes do } \\
\text { atraso ou falha no diagnóstico e/ou tratamento, } \\
\text { 18,4\%; úlceras por pressão, } 18,4 \% \text {; danos de com- } \\
\text { plicações na punção venosa, } 7,7 \% \text {; danos devido a } \\
\text { quedas, 6,2\%; danos em consequência do emprego } \\
\text { de medicamentos, 4,6\%. Eventos adversos evitá- } \\
\text { veis foram responsáveis por } 373 \text { dias adicionais de } \\
\text { permanência no hospital. }\end{array}$ \\
\hline 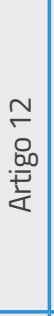 & $\begin{array}{l}\text { Int J Qual } \\
\text { Health Care, } \\
2009\end{array}$ & $\begin{array}{l}\text { Mendes Júnior W, } \\
\text { Martins M, Rozen- } \\
\text { feld S, Travassos C }\end{array}$ & $\begin{array}{l}\text { The assessment of ad- } \\
\text { verse events in hospitals } \\
\text { in Brazil }\end{array}$ & $\begin{array}{l}\text { Coorte re- } \\
\text { trospectivo }\end{array}$ & $\begin{array}{c}\text { A incidência de pacientes com eventos adversos } \\
\text { foi de } 7,6 \% \text {. A proporção geral de eventos adversos } \\
\text { evitáveis foi de } 66,7 \% \text {. A densidade de incidência foi } \\
\text { de } 0,8 \text { eventos adversos por } 100 \text { paciente-dia. A } \\
\text { enfermaria do paciente foi o local mais frequente } \\
\text { dos eventos adversos. Em relação à classificação, } \\
\text { os eventos adversos cirúrgicos foram os mais } \\
\text { frequentes }(35,2 \%) \text {. }\end{array}$ \\
\hline 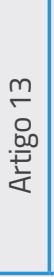 & $\begin{array}{l}\text { Acta Paulista } \\
\text { de Enferma- } \\
\text { gem, } 2013\end{array}$ & $\begin{array}{l}\text { Paranaguá TTB, } \\
\text { Bezerra ALQ, Silva } \\
\text { AEBC, Azevedo } \\
\text { Filho FM }\end{array}$ & $\begin{array}{l}\text { Prevalência de inciden- } \\
\text { tes sem dano e eventos } \\
\text { adversos em uma clínica } \\
\text { cirúrgica }\end{array}$ & Transversal & $\begin{array}{c}\text { Evidenciou-se que } 615 \text { internações foram expos- } \\
\text { tas ao incidente sem dano e } 140 \text { ao evento adver- } \\
\text { so. Dos } 5.672 \text { registros de incidentes, } 218 \text { foram } \\
\text { caracterizados como evento adverso por causarem } \\
\text { dano ao paciente. Os demais não evidenciaram } \\
\text { dano, entretanto apontaram necessidade de ade- } \\
\text { quação dos processos de trabalho. }\end{array}$ \\
\hline \multicolumn{6}{|c|}{ Elaboração: AGUIAR; SALMAZO (2021). } \\
\hline
\end{tabular}

to tempo. Embora a saúde pública tenha avançado de forma significativa nos últimos 30 anos, algumas pesquisas revelam a diminuição da satisfação com a percepção de saúde pessoal ou um aparente descompasso entre o aparato tecnológico de saúde e os parâmetros subjetivos, pautados na autoavaliação do estado de saúde dos usuários nos serviços ${ }^{13-15}$.

Diante disso, quatro fatores que subsidiam a discrepância entre percepção subjetiva e indicadores objetivos de saúde foram propostos, sendo esses: 1) redução das taxas de mortalidade e aumento da prevalência das doenças crônicas não transmissíveis
(DCNT); 2) maior percepção da população sobre os sintomas e doenças, agenciadas por maior conscientização da população sobre saúde; 3) mercantilização generalizada da saúde e a atuação da mídia em disseminar questões de saúde aliadas à criação de um clima de insegurança e alarme sobre a doença, e finalmente, 4) a medicalização progressiva da vida diária, com implicações no desenvolvimento de expectativas não realistas sobre a cura de certas patologias ${ }^{16}$.

O reconhecimento dessa problemática e dos possíveis danos causados pelos cuidados de saúde desencadearam múltiplas reflexões ${ }^{17}$, principalmente, a partir da pu- blicação do documento "Errar é humano: construindo um sistema de saúde mais seguro" pelo Institute of Medicine (IOM), em 1999, que identificou a morte de 44 a 98 mil americanos resultantes de eventos adversos, em grande parte, evitáveis ${ }^{3}$.

Porém, anteriormente, ao longo da história, a segurança do paciente já tem sido abordada como um elemento mínimo para a qualidade do cuidado. Hipócrates, há mais de dois mil anos, enunciou: "primum non nocere", ou seja, "primeiro não ferir". Posteriormente, Florence Nightingale, precursora da Enfermagem Moderna, reforçou: "pode parecer um princípio 
estranho anunciar como requisito básico, que não se deve causar dano ao doente"18.

Parte dessas reflexões e mobilizações foram agenciadas pela constatação de que a ocorrência de eventos adversos envolve custos sociais e econômicos consideráveis, além de implicar em danos irreversíveis aos pacientes e suas famílias. A partir do ano de 2000, a segurança do paciente entrou para a agenda dos pesquisadores de todo o mundo e passou a ser internacionalmente reconhecida como uma dimensão fundamental da qualidade em saúde ${ }^{11}$.

Desse modo, em 2004, a Organização Mundial da Saúde (OMS), reconhecendo a magnitude global do problema da segurança do paciente, lançou a Aliança Mundial para a Segurança do Paciente. O propósito dessa iniciativa foi definir e identificar as prioridades na área de modo a contribuir com uma agenda mundial para a pesquisa no campo. Dentre as questões prioritárias, destacam-se: cuidados de saúde às mães e aos recém-nascidos; cuidados de saúde aos idosos; eventos adversos relacionados a erros de medicação; frágil cultura de segurança, voltada ao processo de responsabilização pelo erro; competências e habilidades inadequadas entre profissionais de saúde; e infecções associadas ao cuidado de saúde ${ }^{19}$.

Assim, a segurança do paciente foi definida pela OMS como a redução do risco de danos desnecessários associados à assistência em saúde até um mínimo aceitável. Esse mínimo aceitável refere-se àquilo que é viável diante do conhecimento atual, dos recursos disponíveis e do contexto em que a assistência foi realizada diante do risco de não tratar ou outro tratamento ${ }^{20}$.

A segurança do paciente é influenciada, apesar dos avanços na área da saúde, pelas iatrogenias cometidas pelos profissionais, as quais refletem diretamente na qualidade de vida dos pacientes, provocando consequências desagradáveis tanto para os pacientes, como para suas famílias, para os profissionais e para o sistema de saúde ${ }^{21}$. Desse modo, a segurança foi a última dimensão incluída de forma explícita no conceito multidimensional de qualidade em serviços de saúde ${ }^{22}$.
Nesse contexto, a qualidade em saúde é compreendida como o grau em que os serviços oferecidos ao paciente diminuem a probabilidade da ocorrência de resultados desfavoráveis e aumentam a probabilidade de resultados favoráveis. Nessa compreensão, os resultados desfavoráveis podem ser compreendidos como os eventos adversos ${ }^{3}$.

Assim, deve-se considerar que a qualidade do atendimento de saúde é a soma de diversos fatores, como: fornecimento de saúde de acordo com o conhecimento científico atual, atendimento direcionado às necessidades dos pacientes, fornecimento adequado de cuidados de saúde de que se é capaz e cuidados que satisfaçam o paciente. Quanto às dimensões que compõem a qualidade do atendimento tem-se a competência profissional ou qualidade técnico-científica, a eficácia, a eficiência, a acessibilidade, a satisfação, a adequação, a equidade e a segurança do paciente ${ }^{13}$.

Diferente das dimensões tradicionais da qualidade, centradas na tomada de decisões certas e oportunas para alcançar resultados em saúde e satisfação dos pacientes, a segurança do paciente tem como foco principal a redução de eventos adversos, mas também de erros, negligências, falhas e omissões do processo assistencial que não causam danos, mas que poderiam ter causado ${ }^{22-23}$.

Dessa forma, os eventos adversos são incidentes passíveis de ocorrer durante a prestação do cuidado à saúde e podem resultar em dano(s) ao paciente que podem ser de natureza física, social e/ou psicológica, o que inclui doença, lesão, sofrimento, incapacidade ou morte ${ }^{24-25}$.

Os eventos adversos são comumente associados ao erro humano individual, mas devem ser considerados como desencadeadores as condições de trabalho, os aspectos estruturais e a complexidade das atividades desenvolvidas. As situações que predispóem ao risco de eventos adversos incluem avanço tecnológico com deficiente aperfeiçoamento dos recursos humanos, desmotivação, delegação de cuidados sem supervisão e sobrecarga de serviço ${ }^{26}$.

Diante disso, os eventos adversos decorrentes da assistência à saúde encon- tram-se presentes nas instituições geralmente devido à sobrecarga de trabalho, muitas vezes, aliada ao dimensionamento de pessoal inadequado e à carência de capacitação dos seus trabalhadores. Além disso, os autores apontam outros fatores, como a comunicação ineficaz, a dificuldade no relacionamento interpessoal entre os profissionais de saúde e o desconhecimento da liderança quanto às fragilidades e potencialidades de sua equipe ${ }^{27}$.

A falta de segurança do paciente causa cerca de 42,7 milhões de eventos adversos com dano ao redor do mundo a cada ano. Desse total, cerca de dois terços acontecem nos países em desenvolvimento e nos países em transiçãa ${ }^{28}$. Segundo a OMS, estima-se que cerca de $10 \%$ dos pacientes sofrem danos relacionados ao cuidado hospitalar em países ocidentais ${ }^{29}$.

No Brasil, ainda há uma elevada incidência de ocorrência de eventos adversos que poderiam ser evitados, conforme resultados demonstrados pelo estudo de Mendes Júnior et al..$^{30}$ : infecções associadas aos cuidados de saúde representaram 24,6\%; complicações cirúrgicas e anestésicas, $20 \%$; danos decorrentes do atraso ou falha no diagnóstico e tratamento, $18,4 \%$; lesões por pressão, $18,4 \%$; danos de complicações na punção venosa, 7,7\%; danos devido a quedas, 6,2\%; danos em consequência do emprego de medicamentos, 4,6\%. Esses eventos, foram responsáveis por 373 dias adicionais de permanência no hospital.

Ainda sobre esse aspecto, outros estudos apontam que a prevalência de eventos adversos no Brasil é em torno de 6 a $18,7 \%^{30-32}$ e a incidência é de $38,4 \% 30$. Entretanto, cerca de $66,7 \%$ desses eventos são considerados evitáveis ${ }^{31}$.

Diante desses dados alarmantes, em 2013 foi instituído no Brasil o Programa Nacional de Segurança do Paciente (PNSP), por meio da Portaria GM/MS n. ${ }^{\circ}$ 529, de 1 de abril de 2013, com a finalidade de apoiar e implementar iniciativas voltadas à segurança do paciente. Nessa mesma direção, a Agência Nacional de Vigilância Sanitária (Anvisa) publicou a Resolução Diretoria Colegiada (RDC) n. ${ }^{\circ}$ 36, de 25 de julho de 2013, na qual estabeleceu ações 
concretas para a promoção do cuidado seguro, a saber: identificação correta do paciente; comunicação efetiva entre os profissionais de saúde; segurança na prescrição, uso e administração de medicamentos; cirurgia segura; higienização das mãos para prevenção de infecções; prevenção de lesão por pressão (LP) e quedas ${ }^{18,25,27,33}$.

Apesar da evolução na abordagem sobre o tema, as investigações sobre eventos adversos em outros níveis de atenção, como a atenção primária à saúde (APS), ainda estão escassas no Brasil. A adoção de estratégias relacionadas à segurança precisa ser mais bem desenvolvida nesse nível de atenção, de forma a identificar quais as oportunidades e os desafios dos profissionais de saúde na prestação de uma atenção à saúde resolutiva, efetiva e de qualidade na $\mathrm{APS}^{11}$.

Por fim, estudo desenvolvido por Andrés et al. ${ }^{34}$ em 48 centros de APS, em 16 comunidades autônomas na Espanha, identificou a prevalência de $11,8 \%$ de eventos adversos, sendo $54,7 \%$ foram considerados leves, $38 \%$ moderados e $7,6 \%$ graves. Nesse sentido, a elevada prevalência de eventos adversos nesse nível de atenção justifica a necessidade de se considerar como prioridade a promoção de medidas para um cuidado seguro também no contexto da APS.

Por fim, a literatura retrata que o con-
A adoção de

estratégias relacionadas

à segurança precisa ser

mais bem desenvolvida

nesse nível de

atenção, de forma

a identificar quais

as oportunidades

e os desafios dos

profissionais de

saúde na prestação de

uma atenção à saúde

resolutiva, efetiva $\mathrm{e}$

de qualidade na APS ceito de segurança do paciente tem evoluído entre os profissionais de saúde, bem como em outras áreas das instituições de saúde hospitalares, inclusive na alta liderança, o que estimula o envolvimento da instituição como um todo ${ }^{35}$, mas que tanto as investigações sobre eventos adversos em outros níveis de atenção, particularmente na APS, quanto a adoção de estratégias relacionadas à segurança do paciente precisam ser mais bem desenvolvidas nesse nível de atençãa ${ }^{11}$.

\section{CONCLUSÃO}

A literatura retrata que o conceito de segurança do paciente tem evoluído entre os profissionais de saúde, bem como em outras áreas das instituições de saúde hospitalares, inclusive na alta liderança, o que estimula o envolvimento da instituição como um todo, mas que tanto as investigações sobre eventos adversos em outros níveis de atenção, particularmente na APS, quanto a adoção de estratégias relacionadas à segurança do paciente precisam ser mais bem desenvolvidas nesse nível de atenção.

Portanto, os resultados deste estudo podem subsidiar discussões entre gestores e profissionais de saúde da APS a fim de identificar as necessidades e limitações para o fomento da segurança do paciente.

\section{REFERÊNCIAS}

1. Gomes ATL, Salvador PYCO, Rodrigues CCFM, Silva MF, Ferreira LL, Santos VEP. A segurança do paciente nos caminhos percorridos pela enfermagem brasileira. Rev Bras Enferm. 2017;70(1):139-46. doi: http://dx.doi.org/10.1590/0034-71672015-0139

2. Alves AS, Aguiar RS. Segurança do paciente no âmbito domiciliar: uma revisão integrativa. Research, Society and Development. 2020;9(3):e181932799. doi: http://dx.doi.org/10.33448/ rsd-v9i3.2700

3. Institute of Medicine. To err is human: building a safer health system. Washington (DC): National Academic Press; 1999.

4. Lemos GC, Azevedo C, Bernanrdes MFVG, Ribeiro HCTC, Menezes AC, Mata LRF. A cultura de segurança do paciente no âmbito da enfermagem: reflexão teórica. Rev Enferm Cent-Oeste Min. 2018;8:e2600. doi: https://doi.org/10.19175/recom. v7i0.2600

5. Moraes CCMS, Aguiar RS. A notificação de eventos adversos e suas lacunas no processo da segurança do paciente. Nursing (São Paulo). 2020;23(271):5025-40. doi: https://doi.org/10.36489/ nursing.2020v23i271p5025-5040

6. Siman AG, Cunha SGS, Brito MJM. Ações de enfermagem para segurança do paciente em hospitais: revisão integrativa. Rev Enferm UFPE On Line. 2017;11(supl. 2):1016-24. doi: https://doi. org/10.5205/reuol.10263-91568-1-RV.1102sup201718

7. Raimondi DC, Bernal SCZ, Oliveira JLC, Matsuda LM. Cultura de segurança do paciente na atenção primária à saúde: análise por categorias profissionais. Rev. Gaúcha Enferm. 2019;40(esp):e20180133. doi: https://doi.org/10.1590/19831447.2019.20180133

8. Aguiar RS, Silva HS. Segurança do paciente na atenção 


\section{artigo}

\section{REFERÊNCIAS}

primária à saúde: uma reflexão teórica. Saúde Coletiva (Barueri). 2020;10(59):4442-55. doi: https://doi.org/10.36489/saudecoletiva.2020v10i59p4442-4455

9. Souza MM, Ongaro JD, Lanes TC, Andolhe R, Kolankiewicz ACB, Magnago TSBS. Cultura de segurança do paciente na atenção primária à saúde Rev Bras Enferm. 2019;72(1):32-9. doi: https:// doi.org/10.1590/0034-7167-2017-0647

10. Aguiar RS, Silva HS, Leandro SS, Oliveira MLC. Percepção dos enfermeiros sobre a cultura de segurança do paciente na atenção primária no contexto da saúde do idoso. Rev Cereus. 2020;12(4):4665. https://doi.org/10.18605/2175-7275/cereus.v12n4p46-65

11. Reis $C T$, Martins $M$, Laguardia J. A segurança do paciente como dimensão da qualidade do cuidado de saúde: um olhar sobre a literatura. Ciên Saúde Colet. 2013;18(7):2029-35. Disponivel em: https:/www.scielo.br/pdf/csc/v18n7/18.pdf

12. Reis CT, Laguardia J, Martins M. Adaptação transcultural da versão brasileira do Hospital Survey on Patient Culture: etapa inicial. Cad Saúde Pública. 2012;28(11):2199-210. Disponível em: https://www.scielo.br/pdf/csp/v28n11/19.pdf

13. Romero MP, González RB, Calvo MSR, Fachado AA. A segurança do paciente, qualidade do atendimento e ética dos sistemas de saúde. Rev Bioét. 2018;26(3):333-42. doi: https:/doi. org/10.1590/1983-80422018263252

14. Rech RS, Hugo FN, Giordani JMA, Passero LG Hilgert JB. Contextual and individual factors associated with dissatisfaction with public emergency health services in Brazil, 2011-2012. Cad Saúde Pública. 2018;34(1):e00175416. doi: https:/doi. org/10.1590/0102-311x00175416

15. Bonadiman RL, Santanna AF, Brasil GA, Lima EM, Lenz D, Endriger DC, Andrade TU. Nível de satisfação dos usuários e verificação do conhecimento dos farmacêuticos em farmácias públicas do Espírito Santo, Brasil. Ciên Saúde Colet. 2018;23(2):627-38. doi: https://doi.org/10.1590/1413-81232018232.03462016

16. Barsky AJ. The paradox of health. N Engl J Med. 1998;318(7):414-8. doi: 10.1056/NEJM198802183180705

17. Rosse FV, Bruijne M, Suurmond J, Essink-Bot ML, Wagner C. Language barriers and patient safety risks in hospital care: A mixed methods study. Int J Nurs Stud. 2016;54:45-53. doi: 10.1016/j.ijnurstu.2015.03.012

18. Reis GAX, Oliveira JLC, Ferreira AMD, Vituri DW, Marcon SS, Matsuda LM. Dificuldades para implantar estratégias de segurança do paciente: perspectivas de enfermeiros gestores. Rev Gaúch Enferm. 2019;40(esp):e10180366. doi: https:/doi. org/10.1590/1983-1447.2019.20180366

19. World Health Organization. Global Priorities for patient safety research. Geneva (Swi): WHO; 2009.

20. World Health Organization. World Alliance for Patient Safety Forward Programme 2008-2009. Geneva (Swi): WHO; 2010.

21. Silva AT, Alves MG, Sanches RS, Terra FS, Resck ZMR. Assistência de enfermagem e o enfoque da segurança do paciente no cenário brasileiro. Saúde Debate. 2016;40(111):292-01. doi: http://dx.doi.org/10.1590/0103-1104201611123

22. Institute of Medicine. Crossing the quality chasm: a new health system for the 21st century. Washington (DC): National Academy Press; 2001.

23. Gama ZAS, Saturno-Hernández PJ, Ribeiro DNC, Freitas MR, Medeiros Pl, Batista AM et al. Desenvolvimento e validação de indicadores de boas práticas de segurança do paciente: Projeto ISEP-Brasil. Cad Saúde Pública. 2016;39(9):e00026215. doi: http://dx.doi.org/10.1590/0102-311X00026215

24. World Health Organization. The International Classification for Patient Safety (ICPS) Taxonomy: more than words. Geneva (Swi); 2009.

25. Brasil. Ministério da Saúde. Portaria n ${ }^{\circ}$ 529, de 1 de abril de 2013. Institui o Programa Nacional de Segurança do Paciente (PNSP). Brasília: Ministério da Saúde; 2013.

26. Beccaria LM, Pereira RAM, Cotrin LM, Lobo SMA, Trajano DHL. Eventos adversos na assistência de enfermagem em uma unidade de terapia intensiva. Rev Bras Ter Intensiva. 2009;21(3):276-82. doi: http://dx.doi.org/10.1590/S0103$507 X 2009000300007$

27. Reis GAX, Hayakawa LY, Murassaki ACY, Matsuda LM, Gabriel CS, Oliveira MLF. Implantação das estratégias de segurança do paciente: percepções de enfermeiros gestores. Texto \& Contexto Enferm. 2017;26(25):e00340016. doi: http://dx.doi. org/10.1590/0104-07072017000340016

28. Jha AK, Larizgoitia I, Audera-Lopez C, Prasopa-Plaizier $\mathrm{N}$, Waters $\mathrm{H}$, Bates $\mathrm{D}$. The global burden of unsafe medical care: analytic modelling of observational studies. BMJ Qual Saf. 2013;22(10):809-15. doi: http://dx.doi.org/10.1136/bmjqs-2012-001748

29. Donaldson LJ. World alliance for patient safety. Geneva (Swi): WHO; 2005.

30. Mendes Júnior W, Pavão ALB, Martins M, Moura MLO, Travassos C. Características de eventos adversos evitáveis em hospitais do Rio de Janeiro. Rev Assoc Med Bras. 2013;59(5):421-8. doi: http://dx.doi.org/10.1016/j.ramb.2013.03.002

31. Mendes Júnior W, Martins $M$, Rozenfeld S, Travassos C. The assessment of adverse events in hospitals in Brazil. Int I Qual Health Care. 2009;21(4):279-84. doi: 10.1093/intqhc/mzp022

32. Paranaguá TTB, Bezerra ALQ, Silva AEBC, Azevedo Filho FM. Prevalência de incidentes sem dano e eventos adversos em uma clínica cirúrgica. Acta Paul Enferm. 2013;26(3):256-62. doi: http://dx.doi.org/10.1590/S0103-21002013000300009

33. Brasil. Agência Nacional de Vigilância Sanitária. Resolução RDC n 36, de 25 de julho de 2013. Institui ações para a segurança do paciente em serviços de saúde e dá outras providências. Brasília: ANVISA; 2013.

34. Andrés JMA, Remón CA, Burillo JV, Solves JJM, Beltrán DO, García ET et al. Estudio APEAS: estudio sobre la seguridad de los pacientes en Atención Primaria de Salud. Madrid (Esp): Ministerio de Sanidad y Consumo; 2007. 